ORIENTAL JOURNAL OF CHEMISTRY

An International Open Free Access, Peer Reviewed Research Journal

www.orientjchem.org
ISSN: 0970-020 X

CODEN: OJCHEG

2018, Vol. 34, No.(1):

Pg. 559-561

(Brief Communication)

\title{
Vibrational Spectra of Copper Tetramesityl Porphyrin Using Vibron Model
}

\author{
J. VIJAYASEKHAR ${ }^{1 *}$, M. V. SUBBA RAO ${ }^{2}$ and V. SREERAM ${ }^{3}$ \\ 'Department of Mathematics, GITAM University, Hyderabad, India. \\ 2Department of Mathematics, ANITS, Visakhapatnam, Andhra Pradesh, India. \\ ${ }^{3}$ Department of Chemistry, A.G \& S.G. Siddhartha Degree College, Vuyyuru, A.P, India. \\ *Corresponding author E-mail: vijayjaliparthi@gmail.com \\ http://dx.doi.org/10.13005/ojc/340165
}

(Received: September 14, 2017; Accepted: December 30, 2017)

\section{ABSTRACT}

In this paper we calculated the fundamental level vibrational spectra of Metalloporphyrin (bio molecule) copper tetramesityl porphyrin (Cu(TMP)) using Vibron model.

Keywords: Vibrational spectra, Vibron model, Metalloporphyrins, $\mathrm{Cu}(\mathrm{TMP})$.

\section{INTRODUCTION}

Group theory is a well known tool that simplifies the process of obtaining a variety of information about molecules and their symmetries. Molecules are classified according to their symmetry properties and from that one can identify, the molecular symmetry point group. The molecular symmetry point group of metalloporphyrins is $D_{4 h}$, which contains the principal $C_{n}$ axis, $n$ perpendicular $\mathrm{C}_{2}$ axis, and the horizontal plane of symmetry.

I-IV are pyrrole rings; 1-8 are substituent positions. $\mathrm{X}$ positions are $(=\mathrm{CH}-)$ bridges

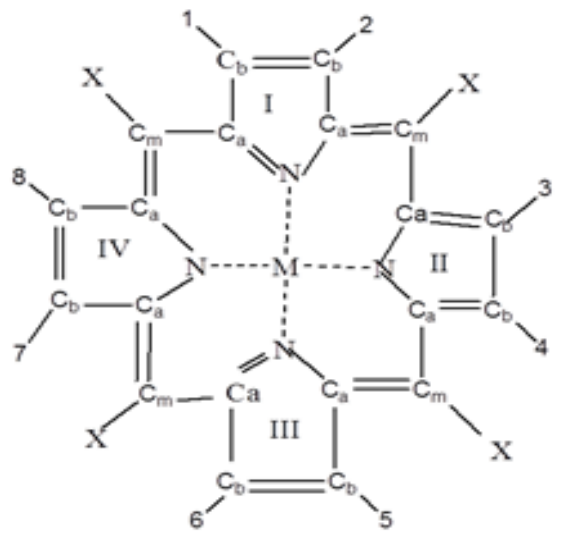

Fig.1. The strucure of metalloporphyrins

This is an Open Access article licensed under a Creative Commons Attribution-NonCommercial-ShareAlike 4.0 International License (https://creativecommons.org/licenses/by-nc-sa/4.0/), which permits unrestricted NonCommercial use, distribution and reproduction in any medium, provided the original work is properly cited. 
In 2008, Karumuri et al., applied Vibron model for coupled anharmonic oscillators to describe the stretching vibrations of medium size molecules and calculated vibrational spectra of nickel octaethyl porphyrins for the stretching mode in $\mathrm{Cm}-\mathrm{H}$. the intervening years extended this model to calculate stretching vibrational frequencies of nickel tetraphenyl porphyrin and copper octaethyl porphyrin for different vibrational bands ${ }^{1-9}$.

\section{Vibron model for Metalloporphyrins}

The general calculation procedure of vibrational spectra of metalloporphyrin by Vibron Model discussed here ${ }^{10,11}$. The Hamiltonian for the polyatomic molecules is of the form Here vary from 1 to $n$ for $n$ stretching bonds and $\left(A_{i}, A_{i j}, \lambda_{i j}\right)$ are algebraic parameters, which are determined by spectroscopic data. Where is an invariant operator (uncoupled bonds) with eigenvalues $-4\left(\mathrm{~N}_{\mathrm{i}} \mathrm{v}_{\mathrm{i}}-\mathrm{v}_{\mathrm{i}}{ }^{2}\right)$ diagonal matrix elements of the invariant operator $\mathrm{C}_{\text {iij }}$ (coupled bonds) and diagonal and non-diagonal matrix elements of Majorana operator $\mathrm{M}_{\mathrm{ij}}$ obtained from the following relations,

$$
\begin{aligned}
& \left\langle N_{i}, v_{i} ; N_{j}, v_{j}\left|C_{i j}\right| N_{i j}, v_{j} ; N_{j}, v_{j}\right\}=4\left[\left(v_{i}+v_{j}\right)^{2}-\left(v_{i}+v_{j}\right)\left(N_{i}+N_{j}\right)\right] . \\
& \left\langle\mathrm{N}_{\mathrm{i}}, \mathrm{v}_{\mathrm{i}} ; \mathrm{N}_{\mathrm{j}}, \mathrm{v}_{\mathrm{j}}\left|\mathrm{M}_{\mathrm{ij}}\right| \mathrm{N}_{\mathrm{i}}, \mathrm{v}_{\mathrm{i}} ; \mathrm{N}_{\mathrm{j}}, \mathrm{v}_{\mathrm{j}}\right\rangle \\
& =\left(\mathrm{N}_{\mathrm{i}} \mathrm{v}_{\mathrm{j}}+\mathrm{N}_{\mathrm{j}} \mathrm{v}_{\mathrm{i}}-2 \mathrm{v}_{\mathrm{i}} \mathrm{v}_{\mathrm{j}}\right) \\
& \left\langle N_{i}, v_{i}+1 ; N_{j}, v_{j}-1\left|M_{i j}\right| N_{i j}, v_{i j} ; N_{j}, v_{j}\right\rangle \\
& =-\left[\mathrm{v}_{\mathrm{j}}\left(\mathrm{v}_{\mathrm{i}}+1\right)\left(\mathrm{N}_{\mathrm{i}}-\mathrm{v}_{\mathrm{i}}\right)\left(\mathrm{N}_{\mathrm{j}}-\mathrm{v}_{\mathrm{j}}+1\right)\right]^{-1 / 2} \\
& \left\langle\mathrm{~N}_{\mathrm{i}}, \mathrm{v}_{\mathrm{i}}-1 ; \mathrm{N}_{\mathrm{j}}, \mathrm{v}_{\mathrm{j}}+1\left|\mathrm{M}_{\mathrm{ij}}\right| \mathrm{N}_{\mathrm{i}}, \mathrm{v}_{\mathrm{i}} ; \mathrm{N}_{\mathrm{j},}, \mathrm{v}_{\mathrm{j}}\right\rangle \\
& =-\left[\mathrm{v}_{\mathrm{i}}\left(\mathrm{v}_{\mathrm{j}}+1\right)\left(\mathrm{N}_{\mathrm{j}}-\mathrm{v}_{\mathrm{j}}\right)\left(\mathrm{N}_{\mathrm{i}}-\mathrm{v}_{\mathrm{i}}+1\right)\right]^{-1 / 2}
\end{aligned}
$$

Where $\mathrm{v}_{\mathrm{i}}(\mathrm{i}=1,2,3, \ldots)$ are vibrational quantum numbers.

The vibron number $N_{i}(i=1,2,3, \ldots)$ for stretching bonds of molecule will be calculated by the following relation

$$
\mathrm{N}_{\mathrm{i}}=\frac{\omega_{i}}{\omega_{i} x_{i}}-2, \mathrm{i}=1,2,3, \ldots
$$

Where $\omega_{\mathrm{e}}$ and $\omega_{\mathrm{e}} \chi_{\mathrm{e}}$ are spectroscopic constants. The initial guess value for the parameter
$A_{i}$ is obtained by using the energy equation for the single-oscillator fundamental mode, which is given as,

$$
\mathrm{E}(\mathrm{v}=1)=-4 \mathrm{~A}_{\mathrm{i}}\left(\mathrm{N}_{\mathrm{i}}-1\right)
$$

Initial guess for $\mathrm{A}_{\mathrm{ij}}$ may be taken as zero. The parameter $\lambda_{i j}$ obtained from the relation

$$
\lambda_{\mathrm{ij}}=\frac{\left|\mathrm{E}_{\mathrm{i}}-\mathrm{E}_{\mathrm{j}}\right|}{a \mathrm{~N}}(a=3,6) .
$$

\section{RESULTS}

Table. 1: Vibrational spectra of $\mathrm{Cu}(\mathrm{TMP})$

\begin{tabular}{lcc}
\hline $\begin{array}{c}\text { Symmetry } \\
\text { Species }\end{array}$ & $\begin{array}{c}\text { Vibrational } \\
\text { mode }\end{array}$ & $\begin{array}{c}\text { Vibrational } \\
\text { frequencies } \\
\left(\mathrm{cm}^{-1}\right)\end{array}$ \\
\hline$A_{1 g}$ & $\left(\mathrm{C}_{\mathrm{m}}-\mathrm{C}\right)$ & 1235.04348 \\
$B_{2 g}$ & $\left(\mathrm{C}_{\mathrm{m}}-\mathrm{C}\right)$ & 1247.03267 \\
$E_{u}$ & $\left(\mathrm{C}_{\mathrm{m}}-\mathrm{C}\right)$ & 1256.90364 \\
$A_{1 g}$ & $\left(\mathrm{C}_{\mathrm{b}}-\mathrm{H}\right)$ & 1470.00431 \\
$B_{2 g}$ & $\left(\mathrm{C}_{\mathrm{b}}-\mathrm{H}\right)$ & 1476.05321 \\
$E_{u}$ & $\left(\mathrm{C}_{\mathrm{b}}-\mathrm{H}\right)$ & 1470.87451 \\
\hline
\end{tabular}

Table. 2: Algebraic parameters

$\begin{array}{lll}\text { Algebraic } & \mathrm{C}_{m}-\mathrm{C} & \mathrm{C}_{\mathrm{b}}-\mathrm{H} \\ \text { parameters } & \end{array}$

\begin{tabular}{lcc}
$A$ & $-2.19234 \mathrm{~cm}^{-1}$ & $-5.90923 \mathrm{~cm}^{-1}$ \\
$A_{i j}$ & $-0.98232 \mathrm{~cm}^{-1}$ & $-2.92012 \mathrm{~cm}^{-1}$ \\
$\lambda_{\mathrm{ij}}(a=3)$ & $0.02421 \mathrm{~cm}^{-1}$ & $0.80834 \mathrm{~cm}^{-1}$ \\
$\lambda_{\mathrm{ij}}(\mathrm{a}=6)$ & $0.20091 \mathrm{~cm}^{-1}$ & $0.03421 \mathrm{~cm}^{-1}$ \\
$\mathrm{~N}$ (Dimensionless) & 140 & 44 \\
\hline
\end{tabular}

\section{CONCLUSION}

In this paper we have calculated the vibrational frequencies of copper tetramesityl porphyrin $(\mathrm{Cu}(\mathrm{TMP}))$ for the stretching modes $\left(C_{m}-C\right)$ and $\left(C_{b}-H\right)$. 


\section{REFERENCES}

1. Karumuri, S. R. Indian J. Phys. 2012, 86(12), 1147-1153

2. Karumuri, S. R.; Sekhar, J.V.; Sreeram, V.; Rao, V. U.M.; Rao, M.V.B.; J Mol Spectrosc. 2011, 269, 119-123

3. Karumuri, S. R.; Srinivas, G.; Sekhar, J.V.; Sreeram, V.; Rao, V. U.M.; Srinivas, Y.; Babu, K.S.; Kumar, V.S.S.; Hanumaiah, A.; Chin Phys B. 2013, 22(9), 090304 (1-8)

4. Karumuri, S. R.; Sarkar, N.K.; Choudhury, J.; Bhattacharjee, R.; Mol. Phys. 2008, 106(14), 1733-1737
5. Karumuri, S. R.; Sarkar, N.K.; Choudhury, J.; Bhattacharjee, R.; Chin Phys Lett. 2009, 26(9), 093301 (1-4)

6. Karumuri, S. R. Chin Phys Lett. 2010, 27(10), $103301(1-4)$

7. Karumuri, S. R. J Mol Spectrosc. 2010, 259, 86-92

8. Karumuri, S R. Eur. Phys. J. 2015, 69, 281

9. Karumuri, S. R.; Sravani, K.G.; Mol. Phys. 2016, 114(5), 643-649

10. lachello, F.; Levine, R. D. Oxford University Press, Oxford. 1995

11. Oss, S. Adv. Chem. Phys. 1996, 93, 455- 649 